\title{
OPTIMIZACIÓN ENERGETICA Y ECONOMICA DE UNA PLANTA DE HARINA DE PESCADO MEDIANTE ANALISIS EXERGETICO Y DETERMINACION EXPERIMENTAL DEL CALOR ESPECÍFICO DE SUS FLUJOS MEZCLA
}

\author{
ENERGY OPTIMIZATION IN A FISH MEAL PLANT USING \\ EXERGY ANALYSIS AND EXPERIMENTAL DETERMINATION \\ OF MIXTURE FLOWS SPECIFIC HEAT
}

\author{
Salome Gonzales Chávez ${ }^{1}$, Ferdinan Estrada Almanza ${ }^{2}$
}

\begin{abstract}
RESUMEN
En el presente trabajo se formula y aplica el análisis exergético al conjunto de elementos consumidores de energía calorifica de una planta típica de harina de pescado, estimando de forma experimental al calor específico de las mezclas sólido-líquido-vapor que conforman la materia prima y el vapor, con el objetivo de optimizar el consumo energético haciendo modificaciones en la instalación original y logrando así la reducción de costos de operación. Generalmente en estas plantas se utiliza el balance energético convencional y la premisa de que los calores específicos corresponden a flujos monofásicos físicos; sin embargo, cuando se presentan flujos mezcla grasa-vapor-agua-sólidos, como es el caso, estos balances simples de energía en cada elemento térmico se hacen débiles y conducen a un alto margen de error en la evaluación energética del conjunto. Dentro de la metodología, los calores específicos de los flujos mezcla grasa-humedad-sólidos-agua se estiman mediante mediciones de indicadores en planta, contrastados con patrones de laboratorio y modelos empírico-matemáticos de autores referentes. Luego se procede a la formulación y cálculo exergético para cada proceso y en conjunto, elaborándose un programa estructurado en Excel tal que calcula de forma vinculada los balances de masa, energía y exergía. A partir de este diagnóstico se elaboran arreglos para seleccionar la mejor alternativa. Como resultados se obtiene el mejor arreglo energético de una planta típica de harina de pescado, esto decir comparado al sistema tradicional: se elimina el exceso de consumo de combustible, se elimina la sobreproducción de vapor $y$, se disminuye las pérdidas de calor en cada elemento del sistema. Ello representa un ahorro anual de diez millones de dólares y además se presentan beneficios adicionales como reducción de emisiones de gases, de efluentes y mejora en la seguridad industrial.
\end{abstract}

Palabras clave.- Optimización, Flujo mezcla, Harina de pescado, Calor especifico, Exergía, Balance, Irreversibilidades.

\section{ABSTRACT}

In this paper we formulate and apply the Exergy Analysis the set heat energy consumption elements of a typical plant of fishmeal, estimating experimentally the specific heat of the solidliquid-vapor mixtures that form the raw material and steam, in order to optimize energy consumption by changes in the original installation and thus achieving the reduction in operating costs. Usually in these plants conventional energy balance and the premise that the specific heats are physical single phase flows is used; however, when solid-fat-water-steam mixture flows are presented, as is the case, these simple energy balances in each heating

La Revista Científica TECNIA protege los derechos de autor bajo la Licencia 4.0 de Creative Commons: Attribution 4.0 International (CC BY 4.0)

${ }^{1}$ Dr. de a Facultad de Ingeniería Mecánica-Universidad Nacional de Ingeniería, ${ }^{2}$ Ing. en Industria Pesquera Diamante-Perú. 
element become weak and lead to a high error margin in energy system assessment. In the methodology, the specific heats of mixture flows fat-water-vapor-solid is estimated by measurement of indicators in the industrial plant, contrasted with laboratory standards and empirical mathematical models. Then proceed to formulation and exergy calculation for each process and together, being elaborated a structured Excel program that calculates balances linked mass, energy and exergy. From this diagnosis suggestions for improvement are made to select the best alternative. As a result the best energy conformation of a typical fishmeal plant is obtained, this mean compared to the traditional system: excess fuel is removed, steam overproduction is removed and heat loss is reduced in each element. This represents an annual savings of ten million dollars plus additional benefits are presented as reduction of greenhouse gas emissions, effluents and improved industrial safety

Key words.- Optimization, Mixed flow, Fishmeal, Specific heat, Exergy, Balance, Irreversibilities.

\section{INTRODUCCIÓN}

Actualmente Perú y Chile procesan el $50 \%$ de la harina de pescado y el $35 \%$ del aceite de pescado en el mundo; siendo Perú el líder mundial en exportaciones de harina y aceite de pescado con una participación del 51\%.

La harina de pescado es un polvo fino obtenido del cocinado, prensado, secado y molido de la materia prima (anchoveta). Es una fuente de alimentación con un alto contenido en proteínas, que es usada como ingrediente en la elaboración de alimentos balanceados para la avicultura, la acuicultura, la ganadería y animales de compañía. Por otro lado, se utiliza como insumo en la elaboración de complementos nutricionales de consumo humano.

En el presente trabajo se realiza la formulación, el diagnóstico y la aplicación en la optimización del uso de la energía en una planta típica de producción de harina de pescado, mediante el Análisis Exergético de los procesos térmicos de consumo de vapor, donde uno de los parámetros de mayor influencia en la cuantificación energética es el calor específico de los flujos mezcla de cada elemento o procesador unitario térmico (cocinas, secadores, evaporadores).

La estimación de los calores específicos de los flujos mezcla se realizan en base a evaluaciones en planta, referencias empíricas y contraste con pruebas de laboratorio. Con estos valores se determinan las entalpías y exergías para así conformar los balances de energía y exergía de cada proceso, que al integrarlos permiten determinar el modelo energético óptimo de una planta.

\section{PLANTEAMIENTO DEL PROBLEMA}

Actualmente el parque industrial peruano de producción de harina de pescado, se caracteriza por los bajos rendimientos de uso del vapor generado a partir de la combustión del petróleo en calderas, esto es pérdidas de calor por fugas, deficientes aislamientos e irreversibilidades en cada uno de sus procesos unitarios, trayendo consigo incrementos en los costos de generación de vapor y por ende en la producción de harina. A ello se suma efectos de contaminación ambiental por emisiones y líquidos calientes que se liberan al medio ambiente.

Como hipótesis se plantea que un adecuado análisis exergético, permite optimizar de forma integrada el consumo energético en una planta de harina de pescado típica referencial.

En un aparato térmico, el análisis exergético evalúa en conjunto: la cantidad de energía que se pierde $o$ se gana (primera ley de la termodinámica), la calidad con que debería aprovecharse o transferirse (segunda ley de la termodinámica) $\mathrm{y}$, el máximo aprovechamiento útil de la temperatura de transferencia respecto del ambiente de referencia (en el caso, la temperatura del medio ambiente) [1].

A diferencia del balance energético convencional, el análisis exergético permite identificar las irreversibilidades de energía en cada proceso unitario de fabricación de harina de pescado, con 
lo cual se determina la energía que no está disponible para ser reutilizada, así como la energía útil potencial que puede recuperarse.

El objetivo del presente estudio es diagnosticar y plantear la optimización del recurso energético en una planta típica de harina de pescado, mediante el análisis exergético de cada proceso térmico de consumo de vapor, estimando los calores específicos de las mezclas de flujo de trabajo.

Una adecuada evaluación exergética de un sistema complejo como es una planta procesadora de harina de pescado, dependerá de la estimación más aproximada del calor específico de las mezclas de trabajo.

Luego se realiza la cuantificación de pérdidas e irreversibilidades que se deben eliminar en un plan de mejoramiento del proceso.

La metodología utilizada para el análisis exergético, se basa en lo siguiente [2 y 3]:

- Identificación del sistema y cada proceso unitario, con división en volúmenes de control.

- Identificación de variables de entrada y variables objetivo.

- Formulación del modelo para la evaluación exergética.

- Estimación de calores específicos de los flujos mezcla del proceso.

- Balance de masa, energía y exergía en cada proceso unitario.

- Calculo de los flujos de exergía, eficiencias exergéticas y destrucción de exergía en cada elemento o aparato térmico.

- Planteamiento de mejoras en el consumo de vapor y distribución energética en cada proceso unitario.

Para la presente investigación, se toma como dominio de estudio a una planta de harina de pescado típica en operación actual, con capacidad de $100 \mathrm{t} / \mathrm{h}$ de procesamiento de pescado, en donde la demanda de vapor es abastecida desde el distribuidor de vapor generado por un conjunto de calderas, y la conformación actual (original) de los aparatos térmicos consumidores de vapor (cocina, secadores, planta evaporadora e intercambiadores de calor auxiliares), se distribuyen según el esquema de la Figura 1.

\section{PROPUESTA DE SOLUCION}

\section{DETERMINACIÓN EXPERIMENTAL DE LOS CALORES ESPECÍFICOS DE LOS FLUJOS MEZCLA}

Uno de los aportes de este trabajo es la determinación experimental de los calores específicos de los flujos mezcla del proceso, conformados por sólido, grasa, líquido y vapor.

Para su cálculo se han tomado las siguientes consideraciones:

- La materia prima y sustancias durante el proceso de fabricación de harina están compuestas por: un porcentaje de sólidos $(\% \mathrm{~S})$, un porcentaje de grasa $(\% \mathrm{G})$ y un porcentaje de humedad $(\% \mathrm{H})$.

- El porcentaje de humedad es la cantidad de agua presente en la materia prima o sustancia.

- El porcentaje de grasa es la cantidad de lípidos presentes en la materia prima o sustancias generadas durante el proceso.

- El porcentaje de sólidos es la suma de proteínas y cenizas (cloruros y sales minerales).

Es decir, la materia prima y las sustancias que se procesan, conforman un flujo mezcla constituido principalmente de tres componentes: grasa, sólidos (proteínas y cenizas) y agua.

Para estimar el calor específico de estas sustancias en conjunto, se toma como referencia el ordenamiento matemático de Choi y Okos [4], cuyos coeficientes se determinan de forma experimental.

Entonces, la fórmula del calor específico se puede ordenar de la siguiente manera:

$$
C_{p}=a \% \text { sólidos }+b \% \text { grasa }+c \% \text { humedad }
$$

Para la estimación de la constante $c$ se toma referencia los modelos de Choi y Okos y Orrego [5], los cuales se muestran en la Figura 2. 

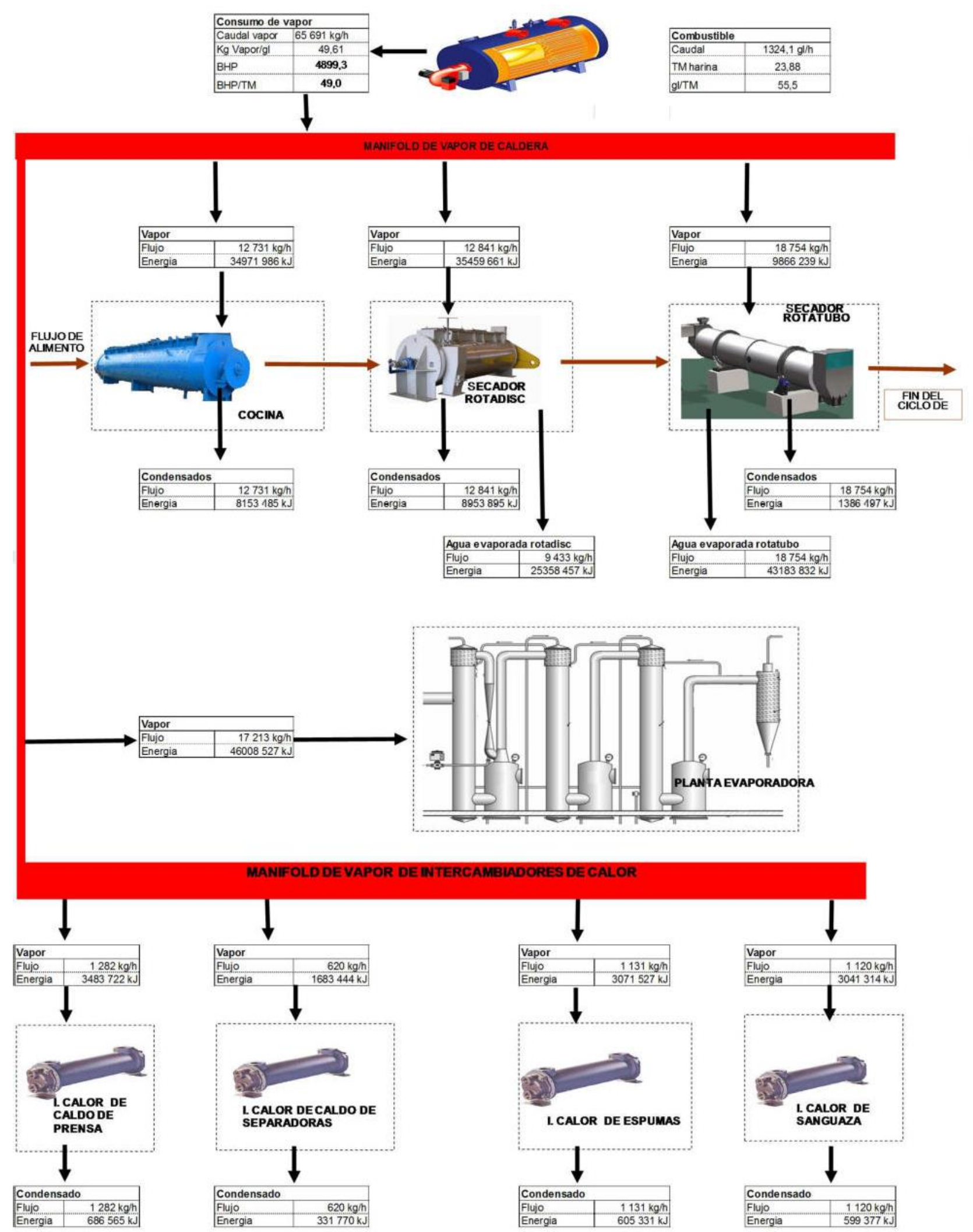

Fig. 1 Esquema térmico actual de la planta de harina de pescado en estudio. 

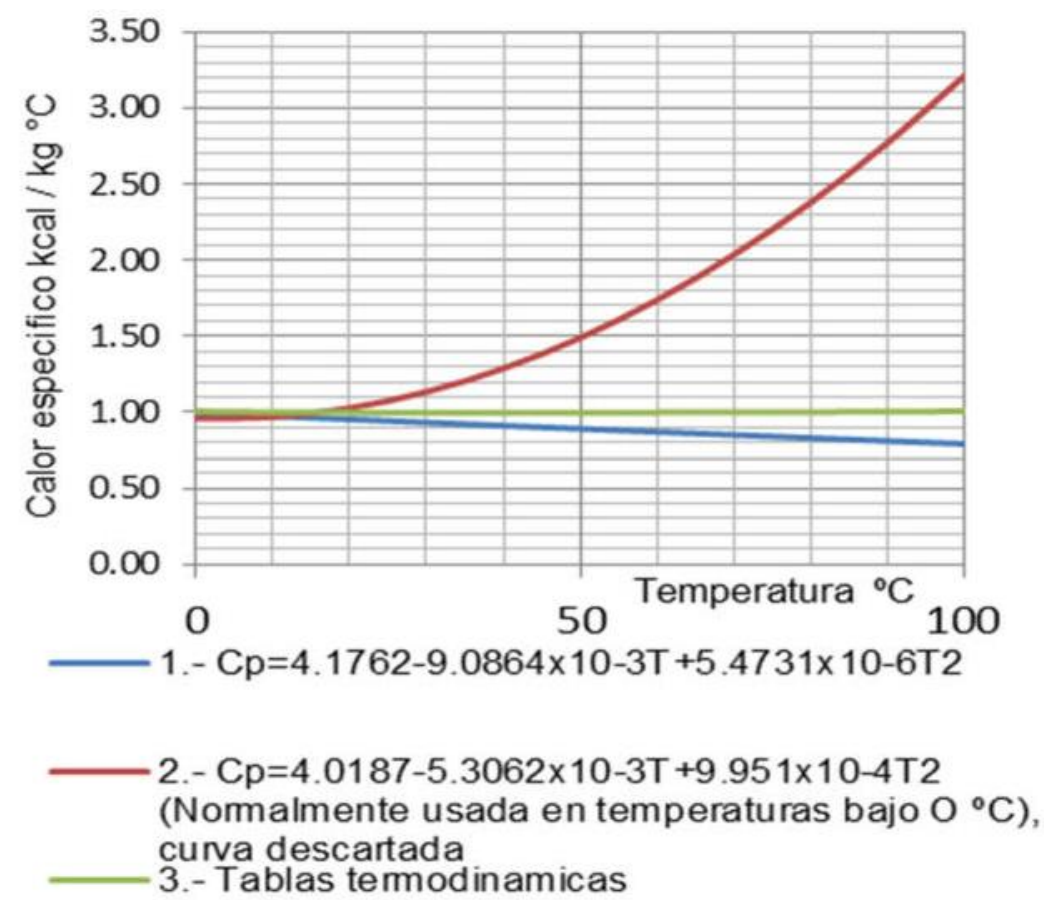

Fig. 2 Calor especifico de la humedad vs temperatura, comparación modelo Choi -Okos y tablas termodinámicas.

De la Figura 2 se observa que el calor específico de la humedad de los modelos 1 y 3 tiene una tendencia parecida comparativamente frente al modelo 2. Por tanto, los valores a considerar del calor especifico de la humedad están entre 0.95 a
1. Para el cálculo del calor específico de las grasas, se toma como referencia los ordenamientos matemáticos empíricos publicados por Alton E. Bayley [6].

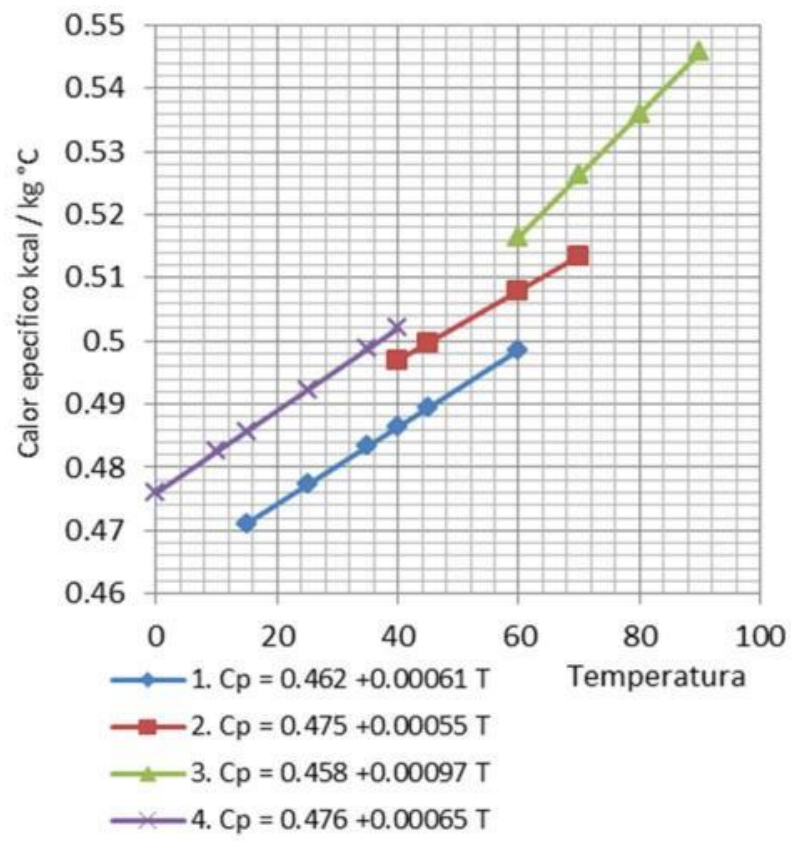

Fig. 3 Variación del calor especifico de las grasas vs temperatura en base al arreglo de Alton E. Bayley. 
De la Figura 3 se tiene que el rango de variación de los cuatro modelos se encuentra entre $0.47 \mathrm{y}$ $1.54 \mathrm{kcal} / \mathrm{kg}{ }^{\circ} \mathrm{C}$. Entonces, para conveniencia de cálculo se considera un valor promedio de calor especifico de las grasas de $0.5 \mathrm{kcal} / \mathrm{kg}{ }^{\circ} \mathrm{C}$.

El componente fundamental, es decir el calor específico de los sólidos en las sustancias mezcla del proceso, se estima tomando como base el ordenamiento matemático de Charm [7], el cual modela al comportamiento del calor específico en función de las proteínas, carbohidratos y cenizas presentes en el proceso. Así, para obtener los valores de estos componentes se realizaron múltiples evaluaciones experimentales realizadas en planta, de proteínas, carbohidratos y cenizas presentes en la harina cuyos resultados se muestran en la Figura 4. Como resultados de este análisis se obtiene un valor promedio para el calor especifico de 0.28 a $0.3 \mathrm{kcal} / \mathrm{kg}{ }^{\circ} \mathrm{C}$.

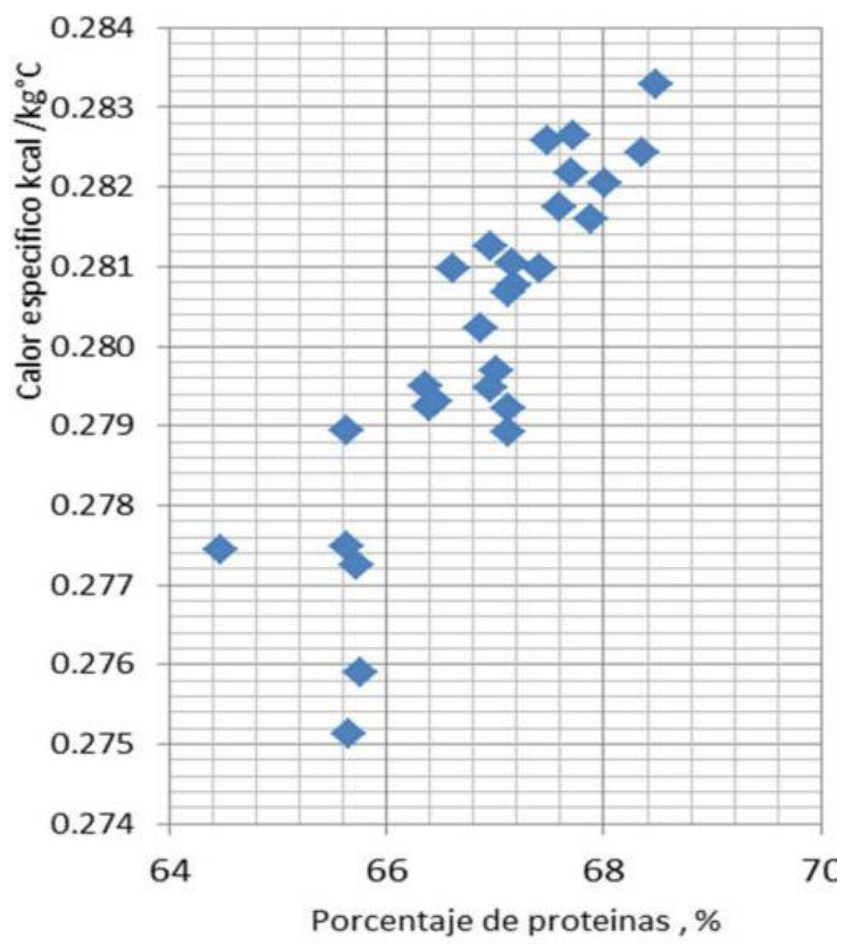

Fig. 4 Variación del calor especifico de los sólidos en función del porcentaje de proteinas.

Para contraste del grado de precisión de los coeficientes obtenidos experimentalmente, se realizaron pruebas muestrales en Laboratorio de la Universidad Nacional de Ingeniería-Perú, cuyos valores obtenidos del calor específico alcanzan una diferencia mínima del orden del $0.1 \%$ respecto al modelo propuesto.

Por tanto, la fórmula para determinar el calor específico [8] de los flujos multifase se puede estimar mediante la siguiente expresión:

$$
C_{p}=0.3 \% S+0.5 \% G+\% H
$$

\section{BALANCE EXERGETICO DE LOS PROCESOS}

El análisis exergético del sistema se realiza a partir del balance de cada elemento térmico del sistema $\mathrm{y}$ en conjunto: cocinador o cocina, secadores, planta evaporadora, intercambiadores de calor. El objetivo es cuantificar y cualificar de forma más exacta respecto a la cuantificación energética convencional, la cantidad de vapor real que consumen cada uno de estos aparatos térmicos y en conjunto, así optimizar tanto la cantidad de vapor a generar como la redistribución apropiada de su consumo en el sistema, consecuentemente logrando el ahorro de combustible en las calderas. 
Para sintetizar la secuencia de cálculo en este artículo, a continuación se muestra la cuantificación exergética de solamente el cocinador o cocina; mientras que en los resultados se da del conjunto.
Entonces, para determinar la cantidad de vapor real consumido durante el proceso de cocción de la materia prima anchoveta, en el análisis se delimita el volumen de control correspondiente a la cocina, tal como se muestra en la Figura 5.

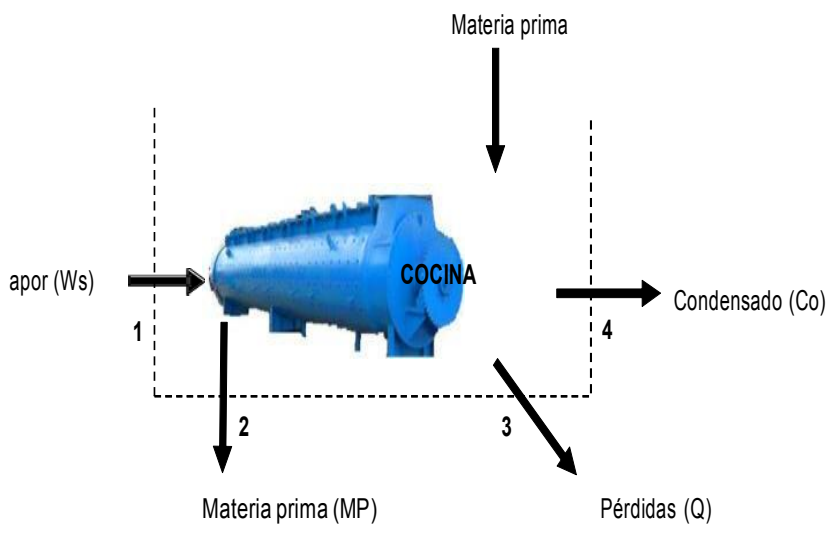

Fig. 5 Volumen de control de la cocina.

El balance simple de energía se realiza aplicando la primera ley de la termodinámica; es decir bajo la expresión siguiente [9 y 10]:

$$
Q+W=M P \cdot h_{2}+C 0 \cdot h_{4}-W S \cdot h_{1}-M P \cdot h_{5}
$$

Para el caso, queda:

$$
Q=M P \cdot\left(\boldsymbol{h}_{\mathbf{2}}-\boldsymbol{h}_{\mathbf{5}}\right)-W s \cdot \lambda
$$

Por tanto, el valor del vapor requerido será:

$$
W s=\frac{M P \cdot C p \cdot\left(T_{2}-T_{5}\right)-Q}{\lambda}
$$

Donde:

Materia prima: $M P$

Masa de vapor: $W_{s}$

Entalpía en el punto i: $h_{i}$

Calor específico: $C_{p}$

Temperatura en el punto i: $T_{i}$

Calor latente del vapor a $\mathrm{T}_{5}: \quad \lambda T_{5}$

Calor cedido por el alimento: $\quad M P C_{p}\left(T_{2}-T_{1}\right)$

Calor por pérdidas: $\quad \mathrm{Q}$

\section{Balance de exergía en el cocinador}

Con el volumen de control correspondiente a la cocina, su balance de exergía se obtiene mediante las ecuaciones:

$$
\begin{gathered}
0=\sum_{j}\left(1-\frac{T o}{T j}\right) \cdot Q+\sum_{e} m_{e} \cdot a_{f e}-\sum_{e} m_{e} \cdot a_{f s}-A d \\
a_{f i}=h-h o-T o \cdot(s-s o) \\
\sum_{e} m_{\theta} \cdot a_{f \theta}=a_{f \theta 1}+a_{f e 5} \\
\sum_{e} m_{s} \cdot a_{f s}=a_{f e 2}+a_{f \theta 3}+a_{f \theta 4}
\end{gathered}
$$

$$
\sum_{j}\left(1-\frac{T o}{T j}\right) \cdot Q=\left(1-\frac{T o}{T_{F}}\right) \cdot Q
$$

Dónde:

$\begin{array}{ll}a_{f e}: & \text { Exergía de entrada } \\ a_{f s:} & \text { Exergía de salida } \\ a_{f:}: & \text { Exergía en el punto i } \\ h: & \text { Entalpía en el punto de frontera del } \\ & \text { volumen de control. En condiciones de } \\ & \text { flujos mezcla, se aproxima al producto } \mathrm{C}_{\mathrm{p}} \\ & \mathrm{T} \\ h_{o}: & \text { Entalpía a la temperatura del ambiente } \\ T_{o}: & \text { Temperatura del ambiente } \\ T_{F}: & \text { Temperatura de la frontera } \\ m_{e}: & \text { Flujos de ingreso al volumen de control } \\ m_{s}: & \text { Flujos de salida del volumen de control } \\ A d: & \text { Exergía destruida } \\ Q: & \text { Pérdidas de calor al ambiente }\end{array}$


En el comportamiento térmico de los procesos, la temperatura de las sustancias varía entre los 15 y $100{ }^{\circ} \mathrm{C}$; en este intervalo el calor específico de la humedad tiene una variación menor al $1 \%$, las grasas menor al $2 \%$ y los sólidos una variación menor al $1 \%$,

De las pruebas realizadas se observa que no hay una variación significativa del calor específico de las grasas, humedad y sólidos debido a la temperatura de trabajo, que se encuentra en un intervalo de 15 a $75{ }^{\circ} \mathrm{C}$. Por lo tanto, el calor específico de las sustancias del proceso solo se verá afectado por la variación de los porcentajes de grasa, humedad y sólidos.

\section{Resultados en el proceso de cocción}

De los resultados obtenidos mediante el balance energético del cocinador, operando en condiciones actuales (ver figura 6), se observa que en flujos entrantes el $88 \%$ de la energía corresponde al vapor y el $12 \%$ a la materia prima (pescado). En flujos salientes, el $79,37 \%$ es ganado por la materia prima, mientras que el calor perdido al ambiente se muestra casi nulo.

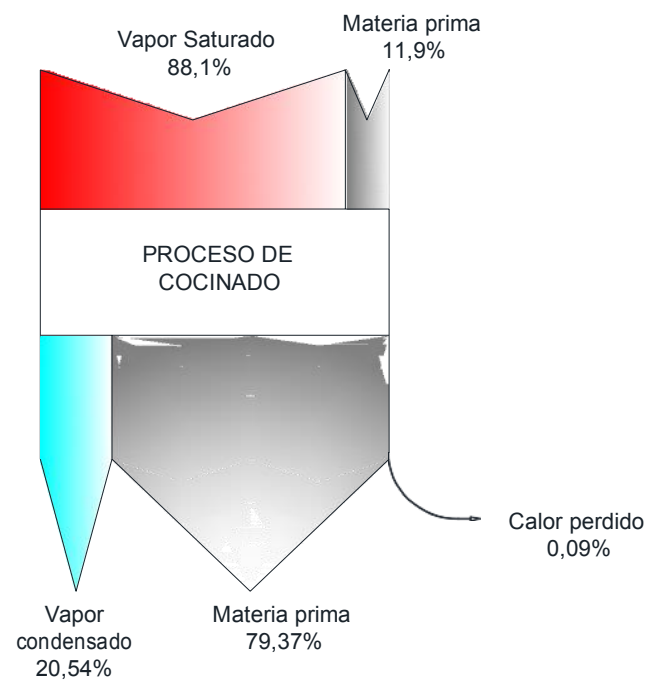

Fig. 6 Balance de energía del proceso de cocción.

Respecto al balance exergético propuesto para la cocina (ver Figura 7), se obtiene que el 99,4\% de la exergía de ingreso representa vapor saturado suministrado por las calderas. De las exergías de salida, el $27,7 \%$ es ganado por la materia prima (pescado), siendo casi nula la exergía de la materia prima de entrada. Por otro lado, se observa que la destrucción de exergía representa aproximadamente el $60 \%$, ello es indicativo de la baja calidad con que se aprovecha el vapor para la transferencia de calor al proceso de cocción (solo el 27.7\%), lo cual significa que la cocina no está adecuadamente seleccionada o diseñada, o que la cantidad de vapor está sobre abastecida. Asimismo, la energía que sale en forma de condensado, puede ser aprovechada antes de regresar al colector de condensados (tanque deareador).

Una forma eficiente y económica de aprovechar el vapor que se está perdiendo en este proceso, es instalar recuperadores de vapor llamados Tanques Flash.

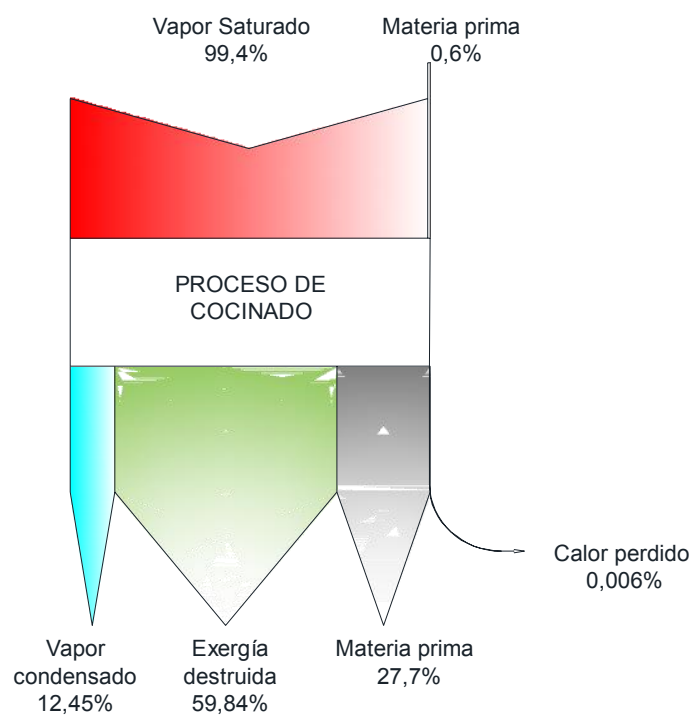

Fig. 7 Diagrama de exergía del proceso de cocción.

Las características del condensado se muestran en la Tabla 1.

Tabla 1. Características del condensado en la cocina.

CONDESADO DE INGRESO (Ci)

\begin{tabular}{ccc}
\hline Flujo de condensado & $\mathrm{kg} / \mathrm{h}$ & 12124 \\
Presión de ingreso & PSI & 60 \\
Entalpía & $\mathrm{kJ} / \mathrm{kg}$ & 2713 \\
Volumen específico & $\mathrm{m}^{3} / \mathrm{kg}$ & 0.0011 \\
\hline
\end{tabular}


Al hacer pasar el condensado por un Tanque Flash se obtendrá vapor útil; así en la Tabla 2 se muestran los resultados, donde se observa que se consigue $627 \mathrm{~kg} / \mathrm{h}$ de vapor Flash, el cual representa una recuperación de energía de más del $5 \%$ respecto al calor de ingreso a la cocina.

Tabla 2. Características del condensado y vapor Flash.

\begin{tabular}{cccccc}
\hline \multicolumn{2}{c}{ CONDESADO DE SALIDA (Cs) } & \multicolumn{5}{c}{ VAPOR FLASH (Vf) } \\
\hline Presión de salida & PSI & 21 & $\begin{array}{c}\text { Presión de } \\
\text { salida }\end{array}$ & PSI & 21 \\
Entalpía & $\mathrm{kJ} / \mathrm{kg}$ & 2240 & $\begin{array}{c}\text { Entalpía } \\
\mathrm{kJ} / \mathrm{kg}\end{array}$ & 2240 \\
Volumen específico & $\mathrm{m}^{3} / \mathrm{kg}$ & 0.001 & $\begin{array}{c}\text { Volumen } \\
\text { específico }\end{array}$ & $\mathrm{m}^{3} / \mathrm{kg}$ & 0.718 \\
Condensado & $\mathrm{kg} / \mathrm{h}$ & 1149 & Vapor flash & $\mathrm{kg} / \mathrm{h}$ & 627 \\
\hline
\end{tabular}

\section{Optimización energética del sistema}

Tal como se muestran en los resultados, los equipos que consumen más energía (vapor) son la cocina, el secador Rotadisc y el secador Rotatubo, los cuales en su conjunto llegan a consumir el
$92 \%$ de todo el vapor que requiere una planta de harina de pescado.

De los balances de exergía, resumidos en la Figura 8 , se observa que del calor que puede recuperarse, se encuentran los condensados y agua evaporada.

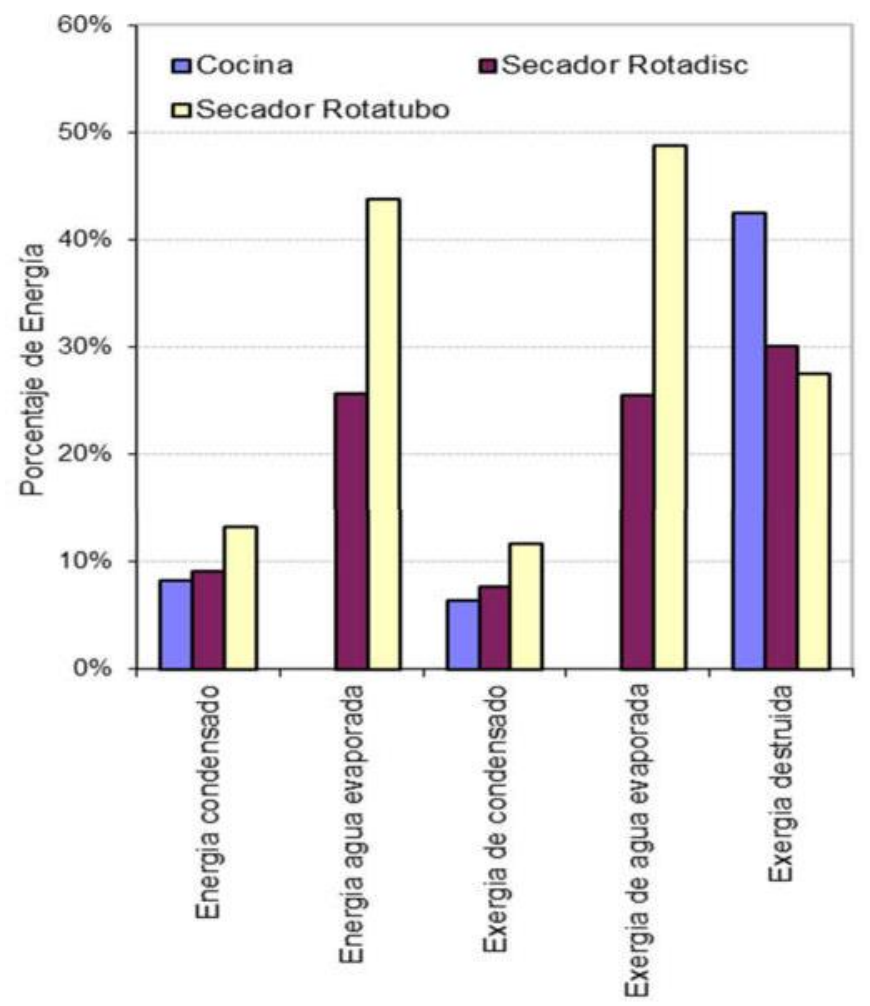

Fig. 8 Porcentajes del 92\% de energía y exergía usada en la planta harinera. 
En relación a la exergía destruida o irreversibilidades, se determina que más del $40 \%$ se realiza en la cocina, alrededor del $30 \%$ en el Secador Rotadisc y $28 \%$ en el Secador Rotatubo. De la energía útil que se puede recuperar, más del $70 \%$ se encuentra en el agua evaporada, la restante se encuentra en el condensado que sale de los equipos térmicos. Entonces, para el proceso de optimización de la energía, se debe maximizar el uso del agua evaporada para calentar las plantas evaporadoras, mientras que el vapor flash del condensado debe maximizarse para calentar los líquidos que resultan del proceso de fabricación de harina de pescado.De la evaluación exergética para la operación y estructura actual de la planta, se puede observar que dentro de los operadores térmicos unitarios, la Cocina alcanza mayores pérdidas de energía, seguido del Secador Rotadisc y el Secador Rotatubo.Como plan de mejoras a las características actuales de instalación integral y operación de la planta en estudio, a partir de la evaluación exergética realizada, se desarrolla un arreglo óptimo de dicha planta, la misma que se ilustra en la Figura 9. 


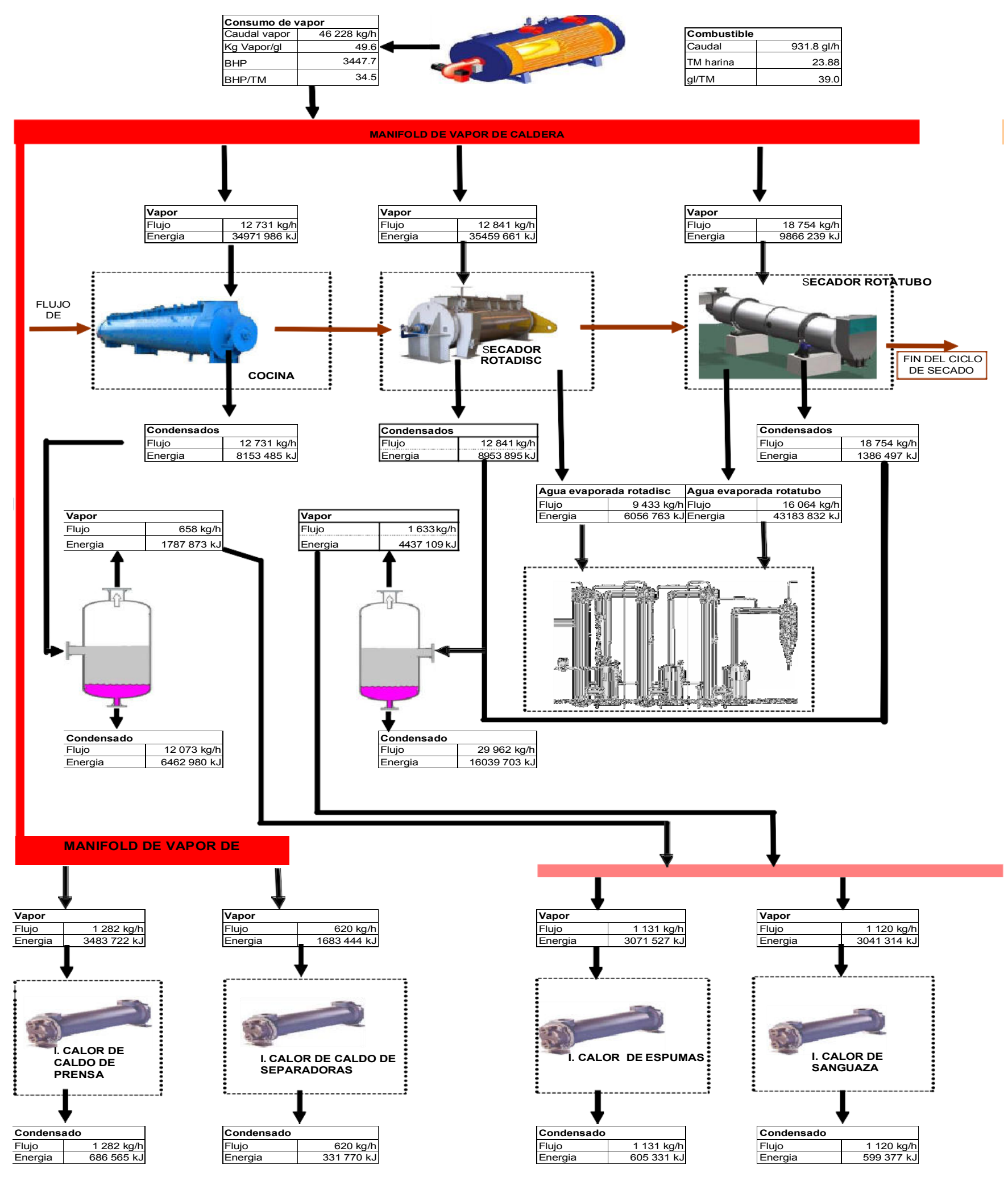

Fig. 9 Esquema térmico de la planta de harina de pescado optimizada. 
En esta última conformación se aprovecha el agua evaporada de los secadores, así como se recupera el condensado de los equipos por medio de tanques flash de los cuales se obtiene vapor que sirve para calentar los líquidos del proceso. El uso del agua evaporada de los secadores y los condensados de retorno, reducen el consumo de vapor de la planta en un $4 \%$ respecto a la instalación original, que está en el orden de 46228 $\mathrm{kg} / \mathrm{h}$. El vapor de consumo optimizado se distribuye en el $96 \%$ en las cocinas y secadores, y el resto está destinado al calentamiento de los líquidos del proceso. En este modelo se genera un ahorro de 16,4 galones de combustible por tonelada de harina respecto a la instalación inicial, lo que equivale a un ahorro de 131 nuevos soles por tonelada de harina.

\section{CONCLUSIONES}

Para temperaturas entre $5^{\circ} \mathrm{C}$ y $100^{\circ} \mathrm{C}$ los calores específicos de la mezcla compuesta por grasas, sólidos y humedad, pueden considerase que no varían con el incremento de temperatura, puesto que los valores experimentales evaluados tienen una variación del $0,1 \%$ respecto al modelo de Charm, los demás modelos comparados arrojan una diferencia promedio de $0,4 \%$. Por lo tanto, el calor específico de las sustancias del proceso solo se verá afectado por la variación de los porcentajes de grasa, humedad y sólidos.

De la evaluación exergética, para la operación y estructura actual de la planta en estudio, se concluye que dentro de los operadores térmicos unitarios, la Cocina alcanza mayores desaprovechamientos de vapor, seguido del Secador Rotadisc y el Secador Rotatubo, ello expresado en el alto porcentaje de exergía destruida, evaluación que no cuantifica un balance energético clásico.

Bajo el balance energético clásico, los elementos que más vapor consumen en una planta de harina de pescado (cocina, secador Rotatubo y secador Rotadisc), muestra que la eficiencia energética de los tres tiene valores similares y del orden de $80 \%$. Mientras que bajo el análisis exergético éstos se diferencian entre el $30 \%$ y $60 \%$ de eficiencia; ello explica que este enfoque no solo cuantifica a la energía transferida sino también cualifica la calidad de aprovechamiento calórico útil en cada unidad consumidora, tomando como referencia el desequilibrio entre un sistema físico y el ambiente que lo rodea.

De la energía útil que se puede recuperar en un proceso de mejora, más del $70 \%$ se encuentra en el agua evaporada, la restante se encuentra en el condensado que sale de los equipos. Entonces en el proceso de optimización, se maximiza el uso del agua evaporada para calentar las plantas evaporadoras, mientras que el vapor flash del condensado se maximiza para calentar los líquidos que resultan del proceso de fabricación de harina de pescado.

\section{REFERENCIAS}

1. Göran Wall, Exergy a useful concept, "Physical Resource Theory Group", Göteborg 1986, 3rd edition.

2. Ibrahim Dincer, Marc A. Rosen., "Exergy, Energy, Environmet and Sustainable Development", first edition 2007.

3. Lozano Serrano, M. A., - Valero Capilla., "Análisis exergético y energético de calderas de vapor", Dpto. de Termodinámica y Físico Química, Escuela Superior de Ingenieros Industriales Universidad de Zaragoza, 2000.

4. Choi, Y., Okos, M. R., "Effects of temperature and composition on the thermal properties of foods", Engineering and Process Applications (M. A. Rao and S. S. H. Rizvi, Editors). Elsevier Applied Science Publishers. Great Britain, London. 1986.

5. Orrego Alate C., "Procesamiento de alimentos", Publicado por Unibiblos - U. Nal. Colombia, 2001.

6. Alton E. Bayley. "Aceites y Grasas Industriales". USA, 1984.

7. Charm, S. E., "Fundamental of Food Engineering". Vna Nostrand Reinhold/ AVI, New Cork. 164 pp. 1971.

8. Dickerson, R. W. Jr., "Thermal properties of food", Chapter 2 in The freezing preservation of food, 1968.

9. Donald Q. Kern, "Procesos de transferencia de calor, Ed McGraw -Hill Book Company INC New York, 1986.

10. Kenneth Wark Jr, Donald E, Richards "Termodinámica", NY, 2001.

Correspondencia: salome@uni.edu.pe

Recepción de originales: enero 2015

Aceptación de originales: abril 2015 